\title{
Angiogenesis inhibitor therapies for advanced renal cell carcinoma: Toxicity and treatment patterns in clinical practice from a global medical chart review
}

\author{
WILLIAM K. OH ${ }^{1,2}$, DAVID McDERMOTT ${ }^{3}$, CAMILLO PORTA $^{4}$, ANTONIN LEVY $^{5}$, REZA ELAIDI ${ }^{6}$, \\ FLORIAN SCOTTE $^{6}$, ROBERT HAWKINS $^{7}$, DANIEL CASTELLANO ${ }^{8}$, JOAQUIM BELLMUNT $^{9}$, \\ SUN YOUNG RHA ${ }^{10}$, JONG-MU SUN ${ }^{11}$, PAUL NATHAN ${ }^{12}$, BRUCE A. FEINBERG ${ }^{13}$, JEFFREY SCOTT ${ }^{13}$, \\ RAY MCDERMOTT $^{14}$, JIN-HEE AHN ${ }^{15}$, JOHN WAGSTAFF ${ }^{16}$, YEN-HWA CHANG ${ }^{17}$, YEN-CHUAN OU ${ }^{18}$, \\ PAUL DONNELLAN $^{19}$, CHAO-YUAN HUANG ${ }^{20}$, JOHN MCCAFFREY ${ }^{21}$, PO-HUI CHIANG ${ }^{22}$, \\ CHENG-KENG CHUANG ${ }^{23}$, CAROLINE KORVES ${ }^{24}$, MAUREEN P. NEARY ${ }^{25}$, \\ JOSE R. DIAZ ${ }^{25}$, FAISAL MEHMUD ${ }^{25}$ and MEI SHENG DUH ${ }^{24}$
}

${ }^{1}$ Dana-Farber Cancer Institute, Boston, MA; ${ }^{2}$ Mount Sinai School of Medicine, The Tisch Cancer Institute, New York, NY; ${ }^{3}$ Beth Israel Deaconess Medical Center, Boston, MA, USA; ${ }^{4}$ IRCCS San Matteo University Hospital Foundation, Pavia, Italy; ${ }^{5}$ Institut Gustave Roussy; ${ }^{6}$ Hôpital Européen Georges Pompidou, Paris, France; ${ }^{7}$ The Christie and University of Manchester, Manchester, UK; ${ }^{8}$ Unit of Uro-Oncology, Department of Medical Oncology, 12 de Octubre University Hospital, I+12 Research Institute, Madrid; ${ }^{9}$ Hospital del Mar - IMIM, Barcelona, Spain; ${ }^{10}$ Severance Hospital, Yonsei Cancer Center, Yonsei University College of Medicine; ${ }^{11}$ Samsung Medical Center, Seoul, Republic of Korea;

${ }^{12}$ Mount Vernon Cancer Centre, Northwood, Middlesex, UK; ${ }^{13}$ P4 Healthcare, Ellicott City, MD, USA;

${ }^{14}$ Adelaide and Meath Hospital, Tallaght, Republic of Ireland; ${ }^{15}$ Asan Medical Center, Seoul, Republic of Korea;

${ }^{16}$ South West Wales Cancer Institute, Singleton Hospital, Swansea, UK; ${ }^{17}$ Taipei Veterans General Hospital, Taipei;

${ }^{18}$ Taichung Veterans General Hospital, Taichung, Taiwan, R.O.C.; ${ }^{19}$ University College Hospital Galway, Galway, Republic of Ireland; ${ }^{20}$ National Taiwan University Hospital, Taipei, Taiwan, R.O.C.;

${ }^{21}$ Mater Misericordiae Hospital, Dublin, Republic of Ireland; ${ }^{22}$ Chang Gung Memorial Hospital, Kaohsiung; ${ }^{23}$ Chang Gung Memorial Hospital, Linkuo, Taiwan, R.O.C.; ${ }^{24}$ Analysis Group Inc., Boston, MA; ${ }^{25}$ GlaxoSmithKline, Collegeville, PA, USA

Received August 12, 2013; Accepted October 9, 2013

DOI: 10.3892/ijo.2013.2181

\begin{abstract}
The aim of this study was to assess the treatment patterns and safety of sunitinib, sorafenib and bevacizumab in real-world clinical settings in US, Europe and Asia. Medical records were abstracted at 18 community oncology clinics in the US and at 21 tertiary oncology centers in US, Europe and Asia for 883 patients $\geq 18$ years who had histologically/cytologically confirmed diagnosis of advanced RCC and received sunitinib $(n=631)$, sorafenib $(n=207)$ or bevacizumab $(n=45)$ as first-line treatment. No prior treatment was permitted. Data were collected on all adverse events (AEs) and treatment modifications, including discontinuation, interruption and dose
\end{abstract}

Correspondence to: Dr Mei Sheng Duh, Analysis Group Inc., 111 Huntington Avenue, Tenth Floor, Boston, MA 02199, USA

E-mail: mduh@analysisgroup.com

Key words: renal cell carcinoma, angiogenesis inhibitors, safety, treatment patterns, interruption, dose reduction reduction. Treatment duration was estimated using KaplanMeier analysis. Demographics were similar across treatment groups and regions. Median treatment duration ranged from 6.1 to 10.7 months, 5.1 to 8.5 months and 7.5 to 9.8 months for sunitinib, sorafenib and bevacizumab patients, respectively. Grade 3/4 AEs were experienced by 26.0, 28.0 and $15.6 \%$ of sunitinib, sorafenib and bevacizumab patients, respectively. Treatment discontinuations occurred in 62.4 (Asia) to $63.1 \%$ (US) sunitinib, 68.8 (Asia) to $90.0 \%$ (Europe) sorafenib, and 66.7 (Asia) to $81.8 \%$ (US) bevacizumab patients. Globally, treatment modifications due to AEs occurred in 55.1,54.2 and 50.0\% sunitinib, sorafenib and bevacizumab patients, respectively. This study in a large, global cohort of advanced RCC patients found that angiogenesis inhibitors are associated with high rates of AEs and treatment modifications. Findings suggest an unmet need for more tolerable agents for RCC treatment.

\section{Introduction}

Given that nearly $25 \%$ of all patients with kidney cancer present with locally advanced or metastatic renal cell carci- 
noma (RCC), kidney cancer is a malignancy with a poor prognosis (1). Conventional therapies, such as chemotherapy and radiation therapy are not effective and only 10 to $20 \%$ of patients benefit from immunotherapy (2-4). Recently enhanced understanding of the etiology of advanced RCC has led to the development of angiogenesis inhibitor agents.

In randomized clinical trials (RCTs), first generation angiogenesis inhibitors sunitinib, sorafenib and bevacizumab plus interferon $\alpha$, have demonstrated efficacy in prolonging progression-free survival and/or overall survival as first-line treatment (4-6). The efficacy of sunitinib and sorafenib has also been established in expanded access programs (EAP) $(7,8)$. Due to their strong efficacy profiles, these agents have become the new standard of treatment for advanced RCC. All these drugs were approved by corresponding regulatory agencies for use in the US, Europe and Asia (9-13).

However, RCTs and EAPs have also demonstrated that these angiogenesis inhibitors are associated with high rates of toxicity and treatment modifications, including discontinuations and dose changes. Since clinical trials may not be representative of real-life clinical practice due to treatment selection criteria, observational studies are necessary to understand the effects of treatment in the wider population of patients who actually receive these therapies. Small observational studies conducted in real-world clinical practice settings in US, Korea, Japan and Europe have provided further evidence of high toxicity profiles associated with these agents (14-21). Data from these varied care settings highlight that adverse events (AEs) in advanced RCC patients receiving angiogenesis therapies are common and often lead to treatment modifications, including treatment discontinuation.

As the use of angiogenesis inhibitors rises over time and treatment paradigms continue to evolve, there is a critical need to gain a thorough understanding of toxicity profiles and treatment patterns of these agents across various realworld clinical settings. Therefore, the goal of this study was to examine the toxicity profiles of sunitinib, sorafenib, and bevacizumab in advanced RCC among patients treated in US, Europe and Asia, and describe how clinicians in these settings modify treatment according to patient experiences.

\section{Materials and methods}

Study design. A retrospective study was conducted using data from medical records for eligible patients with advanced RCC who received anti-angiogenic therapies. The observation period for each patient started from the date of first angiogenesis inhibitor prescription or administration to the earliest of date of death, last follow-up date at the clinic or date of medical record abstraction. Data on second-line angiogenesis inhibitor treatment were also abstracted. The study drugs sunitinib, sorafenib and bevacizumab are manufactured by Pfizer, Bayer Healthcare Pharmaceuticals, and Hoffmann-LaRoche Inc., respectively.

Study population. To become eligible in the study patients were required to meet the following inclusion criteria: i) have had a confirmed histological and/or cytological diagnosis of locally advanced or metastatic RCC; ii) 18 years old or older at the time of confirmed diagnosis of advanced RCC; and iii) received at least 1 dose of oral sunitinib or sorafenib or intravenous (IV) administration of bevacizumab with or without interferon, after January 1, 2005. Previous immunotherapy or chemotherapy was not allowed. Patients were excluded if their first angiogenesis inhibitor treatment was initiated less than three months prior to the start date of medical record data abstraction, which varied across sites, to ensure adequate follow-up time.

Data source. Medical records for eligible patients were retrospectively abstracted by the clinical staff at 18 community oncology clinics in the US, and at 21 tertiary oncology centers across US $(n=2)$, Europe $(n=11$; France $n=2$, Ireland $n=3$, Italy $\mathrm{n}=1$, Spain $\mathrm{n}=2$ and UK $\mathrm{n}=3)$, and Asia $(\mathrm{n}=8$; Korea $\mathrm{n}=3$ and Taiwan $n=5$ ). Data collected included date of RCC diagnosis, sociodemographic information, comorbidities, prior radiological treatments, metastatic site(s), baseline Eastern Cooperative Oncology Group (ECOG) performance status, dates and doses of anti-angiogenesis therapies prescribed or administered, reasons for changes in anti-angiogenesis therapies, and information on AEs. Other key data elements abstracted included the first and last dates of sunitinib, sorafenib and bevacizumab treatments, treatment modifications, and baseline and follow-up tumor measurements. Data were collected using a web-based case report form (CRF) created for this study. Data collection for this study spanned from July, 2007 through May, 2011. This study was approved in all centers by the ethics committees for tertiary oncology clinics and the New England Institutional Review Board for oncology community clinics in the US.

\section{Outcome definitions}

Assessment of toxicity. All toxicity was analyzed retrospectively according to the experience recorded by investigators in daily clinical practice. AEs were graded using the National Cancer Institute Common Terminology Criteria for AEs (CTCAE) version 3.0. (22). If the severity of the AE was unknown then grade 1 was assigned. Only AEs experienced by patients during their first-line angiogenesis inhibitor treatment were considered for the assessment of safety.

Assessment of treatment patterns. Treatment modifications that occurred during first-line angiogenesis inhibitor treatment were examined. Reasons for treatment modifications were also abstracted from patients' medical records, if available. Treatment modifications considered were treatment discontinuation, treatment interruption (temporary stoppage of treatment with intent to resume treatment), dose reduction and dose increase. Patterns of switching between different angiogenesis inhibitors to second-line treatment were also examined, including reasons for switching.

Treatment duration. The duration of first-line treatment extended from the date of initiation of treatment to the date of treatment end, death, or last follow-up, whichever occurred first. Patients who did not discontinue their treatment were censored at the last follow-up.

Statistical analysis. Descriptive statistics were used to characterize baseline patient characteristics and report AE occurrences, and treatment patterns. Means and medians were used to describe continuous variables while frequen- 


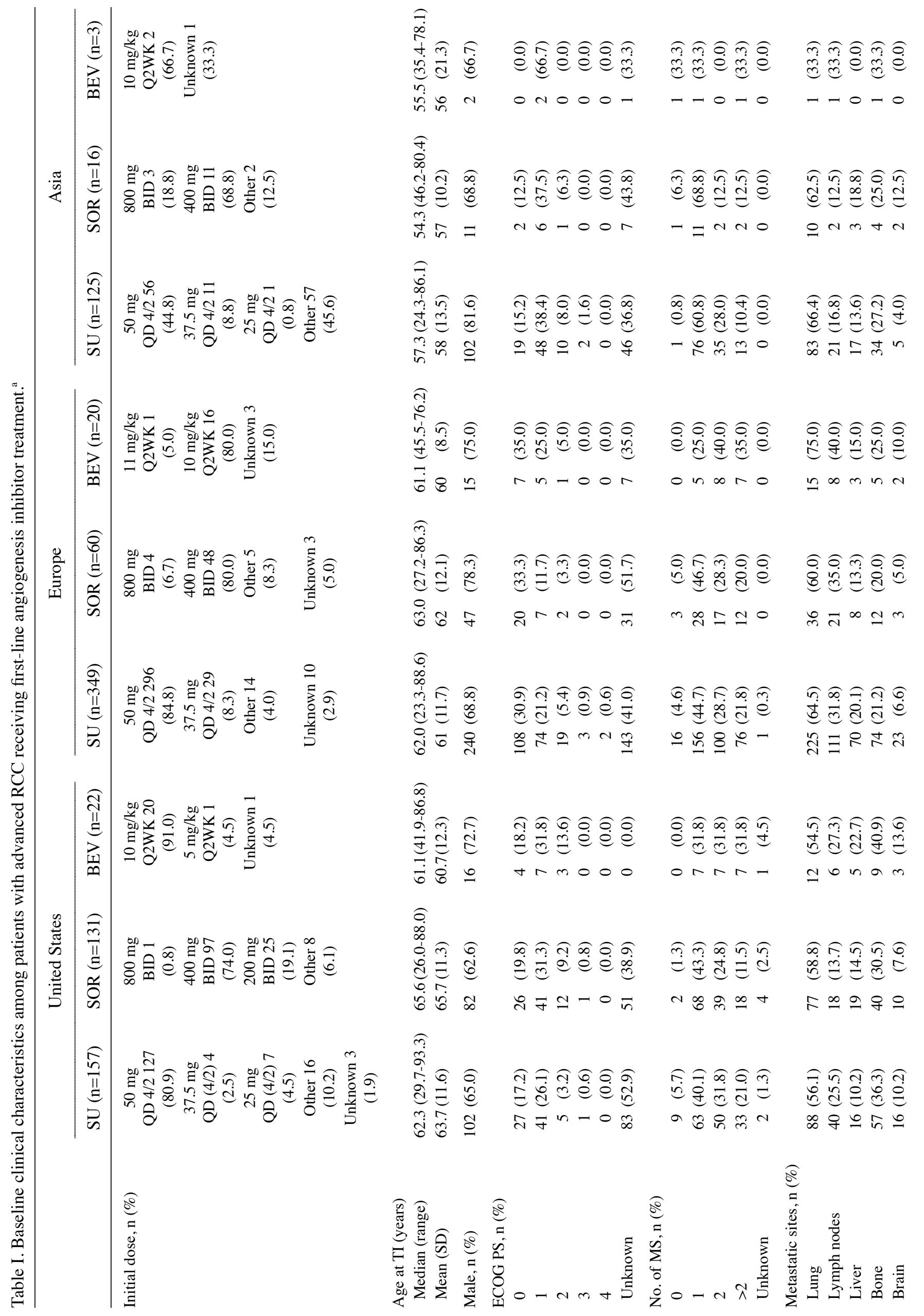


cies and proportions were used to describe categorical variables. The Kaplan-Meier survival analysis method was used to calculate median treatment duration and account for censoring. The corresponding 95\% confidence intervals (CI) were calculated using the log transformation method. All analyses were performed using SAS software version 9.2 (SAS Institute Inc., Cary, NC, USA).

\section{Results}

Patient characteristics. Table I presents the baseline characteristics of the patients. A total of 883 patients satisfied the eligibility criteria, including 157 (US), 349 (Europe), and 125 (Asia) patients treated with sunitinib; 131 (US), 60 (Europe), and 16 (Asia) patients treated with sorafenib; and 22 (US), 20 (Europe), and 3 (Asia) patients treated with bevacizumab. Most patients across the three geographical regions initiated treatment on recommended dosing: $50 \mathrm{mg}$ QD 4/2 for sunitinib [range: $44.8 \%$ (Asia) to $84.8 \%$ (Europe)], $400 \mathrm{mg}$ BID for sorafenib [range: $68.8 \%$ (Asia) to $80.0 \%$ (Europe)], and $10 \mathrm{mg} / \mathrm{kg}$ Q2WK for bevacizumab [range: 66.7\% (Asia) to $91.0 \%$ (US)].

Toxicity profile. Table II presents the rates of all grade and grade 3/4 AEs. The proportion of sunitinib patients experiencing at least one AE was about $87 \%$ across all regions. Among patients receiving sorafenib, $78.3 \%$ (Europe) to $87.8 \%$ (US) experienced at least one AE, and among patients receiving bevacizumab, $33.3 \%$ (Asia) to $77.3 \%$ (US) experienced at least one AE. Specific AEs experienced by at least $5 \%$ of patients in at least one treatment group are reported. The three most common all grade AEs in patients treated with sunitinib were fatigue/asthenia [range: $18.4 \%$ (Asia) to $58.5 \%$ (Europe)], mucositis/stomatitis [range: $22.9 \%$ (US) to $42.1 \%$ (Europe)] and diarrhea [range: $17.6 \%$ (Asia) to $34.4 \%$ (US)]. Patients treated with sorafenib commonly experienced the following all grade AEs: fatigue/asthenia [range: $6.3 \%$ (Asia) to $39.7 \%$ (US)], diarrhea [range: $6.7 \%$ (Asia) to $35.1 \%$ (US)], and nausea [range: $5.0 \%$ (Europe) to $23.7 \%$ (US)]. Among patients who received bevacizumab, the most common all grade AEs reported on the medical charts were fatigue/asthenia [up to $45.4 \%$ (US)] and proteinuria [up to $22.7 \%$ (US)].

Treatment patterns. Table III summarizes treatment patterns for first-line treatment with angiogenesis inhibitor. Median treatment duration, in months, was 6.1 (US), 10.7 (Europe) and 10.7 (Asia) for sunitinib; 5.1 (US), 8.5 (Europe) and 7.1 (Asia) for sorafenib; and 9.2 (US), 9.8 (Europe) and 7.5 (Asia) for bevacizumab. Treatment discontinuation occurred in $62.4 \%$ (Asia) to $63.1 \%$ (US) of patients treated with sunitinib, $68.8 \%$ (Asia) to $90.0 \%$ (Europe) of patients treated with sorafenib, and $66.7 \%$ (Asia) to $81.8 \%$ (US) of patients treated with bevacizumab. Reasons for treatment modifications were available at all but one site. Among sites with these data, progressive disease was the most commonly recorded reason for treatment discontinuation [sunitinib, 33.1\% (US) to $40.0 \%$ (Asia); sorafenib, $42.0 \%$ (US) to $55.6 \%$ (Europe); bevacizumab, $33.3 \%$ (Asia) to $46.7 \%$ (Europe)] followed by AEs [sunitinib, $18.4 \%$ (Asia) to $23.6 \%$ (US); sorafenib, 


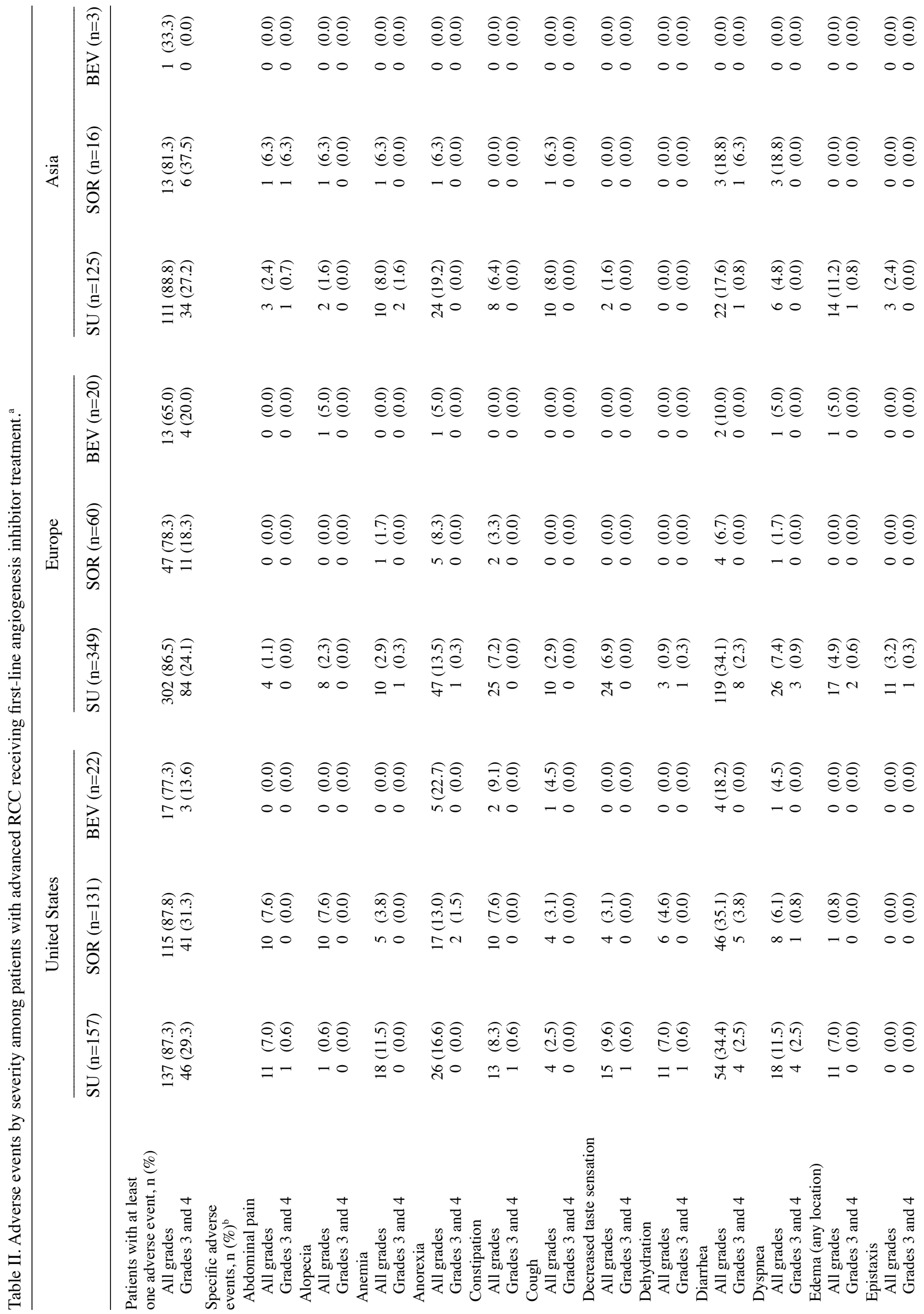




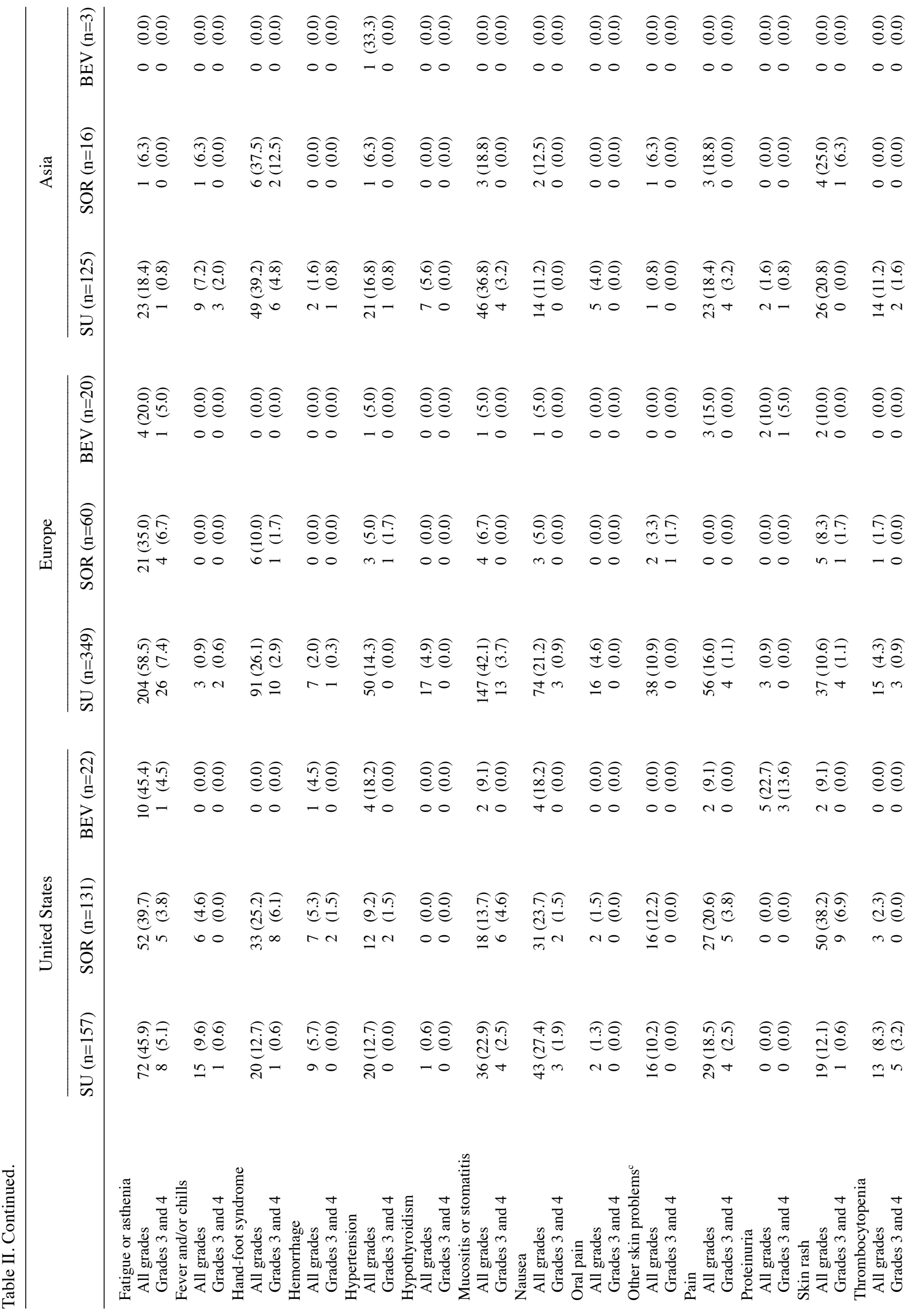


$6.3 \%$ (Asia) to $28.2 \%$ (US); bevacizumab, $0 \%$ (Asia) to $27.3 \%$ (US)]. Drug dosage was reduced in $34.4 \%$ (US) to $48.0 \%$ (Asia) of patients treated with sunitinib, and $21.7 \%$ (Europe) to $45.8 \%$ (US) of patients treated with sorafenib. AEs were the most commonly reported reason for reduced dosage for sunitinib [29.2\% (US), 37.1\% (Europe), and 36.8\% (Asia)], and sorafenib [43.5\% (US), 20.4\% (Europe), and $31.3 \%$ (Asia)]. After the discontinuation of first-line treatment, $20.4 \%$ (US) to $24.0 \%$ (Asia) of patients treated with sunitinib, $31.3 \%$ (Asia) to $50.0 \%$ (Europe) of patients treated with sorafenib, and $59.1 \%$ (US) to $70.0 \%$ (Europe) of patients treated with bevacizumab received second-line therapy.

Table IV describes specific AEs reported as reasons for first-line treatment modifications. Among the AEs of interest, vomiting was the most common AE leading to discontinuation of first-line sunitinib in the US (21.6\%), fatigue/asthenia was most common in Europe (32.7\%), and mucositis or stomatitis was the most common reason in Asia (34.8\%). For sorafenib, skin rash most commonly led to treatment discontinuation in the US (27.8\%); diarrhea and hand-foot syndrome most commonly led to discontinuation in Europe (37.5\%). The most common AEs reported as reason for dose reduction for sunitinib varied across regions: diarrhea in US (25.5\%), fatigue in Europe (31.5\%) and mucositis or stomatitis in Asia (26.1\%). Skin rash was the most common AE for dose reduction among sorafenib patients in US (31.6\%), and hand-foot syndrome was the most common reason in Europe (44.4\%).

\section{Discussion}

Findings from the current study with data from 883 patients contribute to the growing body of knowledge on the use of sunitinib, sorafenib and bevacizumab as first-line agents among patients with advanced RCC treated in real-world clinical practice across different geographical regions. Tolerability and management of side-effects for patients receiving sunitinib, sorafenib and bevacizumab as first-line anti-angiogenesis treatment for advanced RCC are significant issues for patients and the physicians who care for them.

There were variations in treatment and outcomes across global regions. In the US and Europe, patients receiving sunitinib were almost twice as likely as those in Asia to initiate therapy at $50 \mathrm{mg}$ QD 4/2. This observation raises the issue relative to the use of fixed doses of suntinib (and also of all molecularly targeted agents), irrespective of parameters like gender and body weight/body surface area. Indeed, a population pharmacokinetic analysis identified low body weight (and female gender) as covariates that significantly increase exposure to sunitinib, potentially leading to increased toxicity (23). Moreover, the most commonly experienced AEs also varied across region. In the US and Europe, fatigue was the most common AE among patients receiving sunitinib, sorafenib and bevacizumab while hand-foot syndrome was the most common AE in Asian patients receiving sunitinib or sorafenib. Notably, the AEs most commonly leading to treatment discontinuation also varied.

Despite these differences, there were some universal findings across settings. Notably, the median treatment duration was generally shorter in this observational study compared 


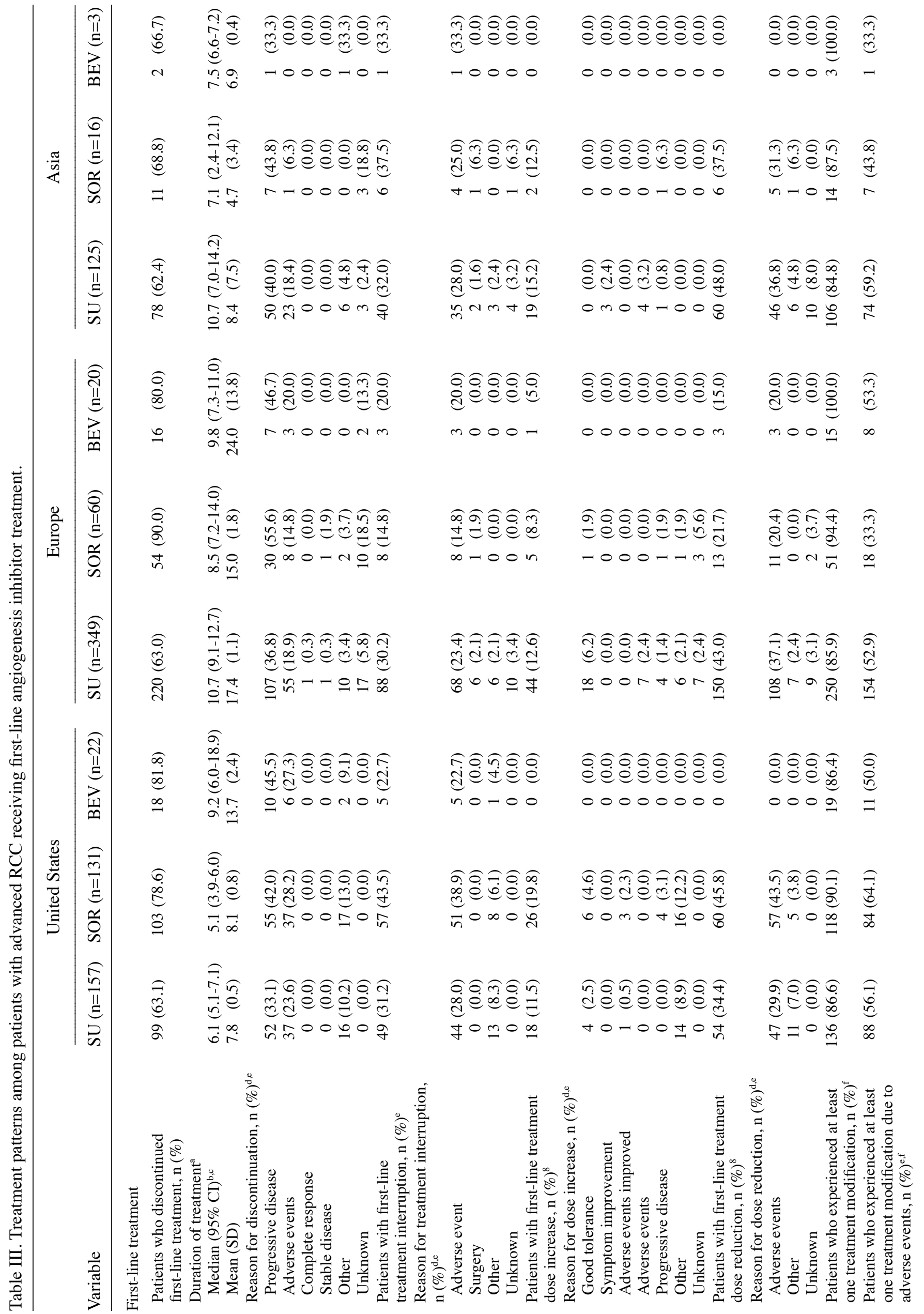


with that reported in RCTs and EAPs. The median treatment duration for sunitinib was 11 and 16.6 months in the RCT and EAP $(4,7)$, respectively, whereas the median ranged from 6.1 to 10.7 months across regions in this study. Similarly, the median treatment duration for sorafenib was 12 months in the EAP (8) whereas it ranged from 5.1 to 8.5 months across regions in the current analysis. For bevacizumab the median treatment duration was 9.7 months in one RCT (24) and 8.2 months in another RCT (25) whereas it ranged from 7.5 to 9.8 months across regions in this study.

Treatment discontinuation was high, reaching $63.1 \%$ among sunitinib patients in the US, $90.0 \%$ among sorafenib patients in Europe, and $81.8 \%$ of bevacizumab patients in US. For patients who discontinued sunitinib treatment due to AEs the average number of AEs per discontinuation was 2.3-2.5 across regions; for sorafenib it was 1.9-3.1. This illustrates that in real-world practice physicians manage multiple AEs per patient and may discontinue or modify treatment based on the observed effects of these AEs. The proportion of patients with any type of treatment modification due to an AE was also consistently high across all regions, reaching 59.2\% among sunitinib patients in Asia, $64.1 \%$ among sorafenib patients in US and $53.3 \%$ of bevacizumab patients in Europe.

This study builds upon prior evidence from RCTs and EAPs on the toxicity and treatment patterns of angiogenesis inhibitors. Similar to results in this global chart review study, treatment discontinuation was high in RCTs and EAPs. In the RCT comparing sunitinib to interferon $\alpha, 86 \%$ of patients experienced a treatment discontinuation (versus 62.4 to $63.1 \%$ in the current study) (4). In the EAP for sorafenib, $100 \%$ of patients experienced a treatment discontinuation (versus 68.8 to $90.0 \%$ in the current study) (8). In the RCT comparing bevacizumab plus interferon to placebo, $72 \%$ of patients experienced a treatment discontinuation (versus 66.7 to $81.8 \%$ in the current study) (24). In RCTs, diarrhea was the most commonly reported AE for both sunitinib and sorafenib (versus fatigue in the US and Europe, and hand-foot syndrome in Asia in the current study) and anorexia was the most commonly reported $\mathrm{AE}$ for bevacizumab (versus fatigue in the current study for US and Europe) $(4,8,24)$. In EAPs for sunitinib and sorafenib, the most commonly reported AEs were diarrhea and hand-foot syndrome.

Differences with RCT may arise due to differences in the underlying study populations as well as how data are collected. For example, the proportion of patients with brain metastasis in the current study were 7.0, 7.2 and $11.1 \%$, for sunitinib, sorafenib and bevacizumab, respectively, while these patients were excluded in RCTs. Besides, RCTs have well-defined operational definitions for AE identification and gradation, as well as a rigorous protocol to capture them whereas in a retrospective setting such as in the current study, AEs are captured based on the treating physicians' reports and judgments. Sometimes physicians may record only those AEs that lead to a treatment modification or if the AEs are severe enough to warrant specific treatment; therefore, underreporting of AEs in this study may have occurred.

Findings from this study were generally consistent with those from other observational studies (14-20). In the Korean study by Hong et al (14), 76\% of sunitinib patients had a dose interruption or dose reduction due to AEs, and $11 \%$ overall 


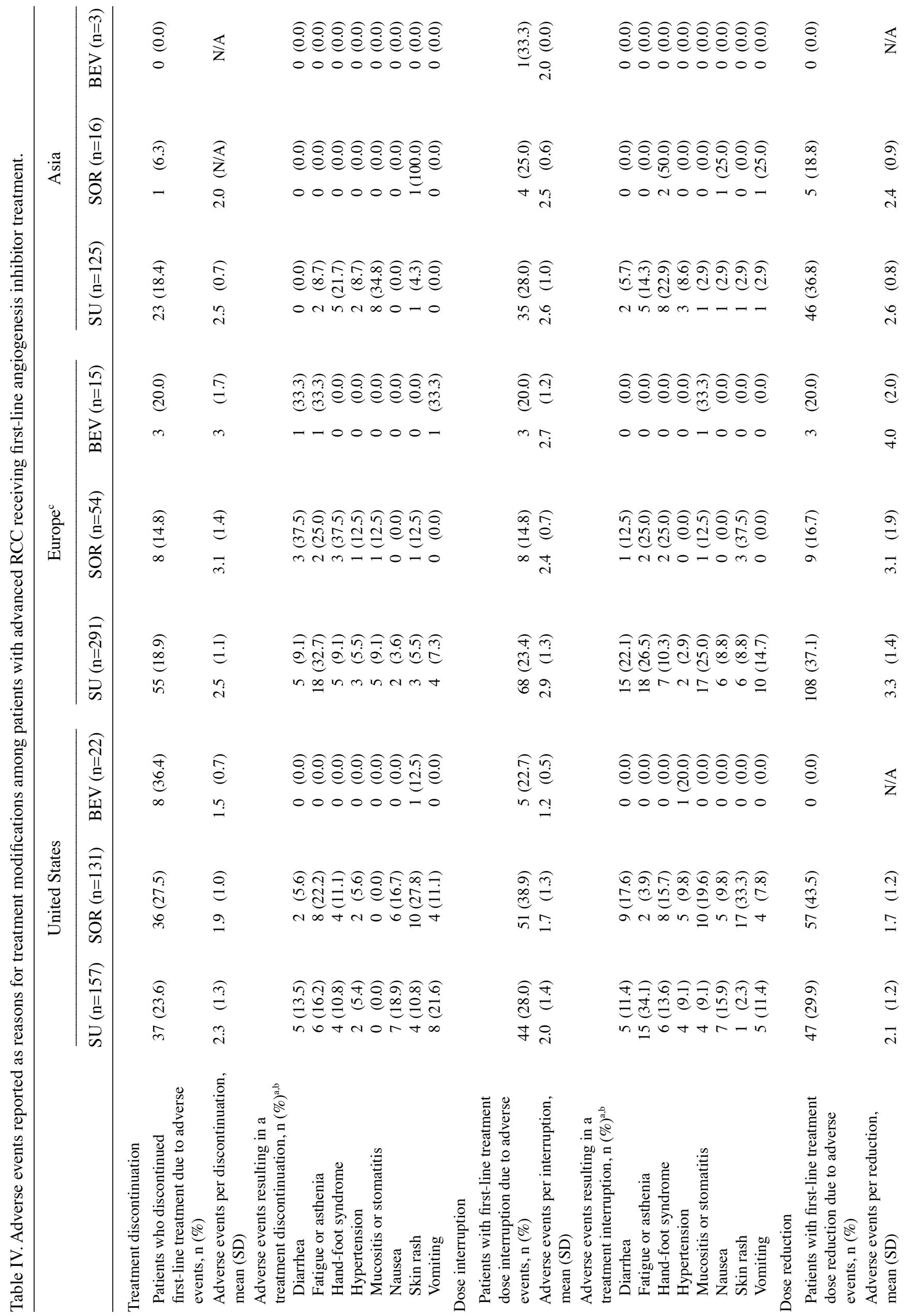




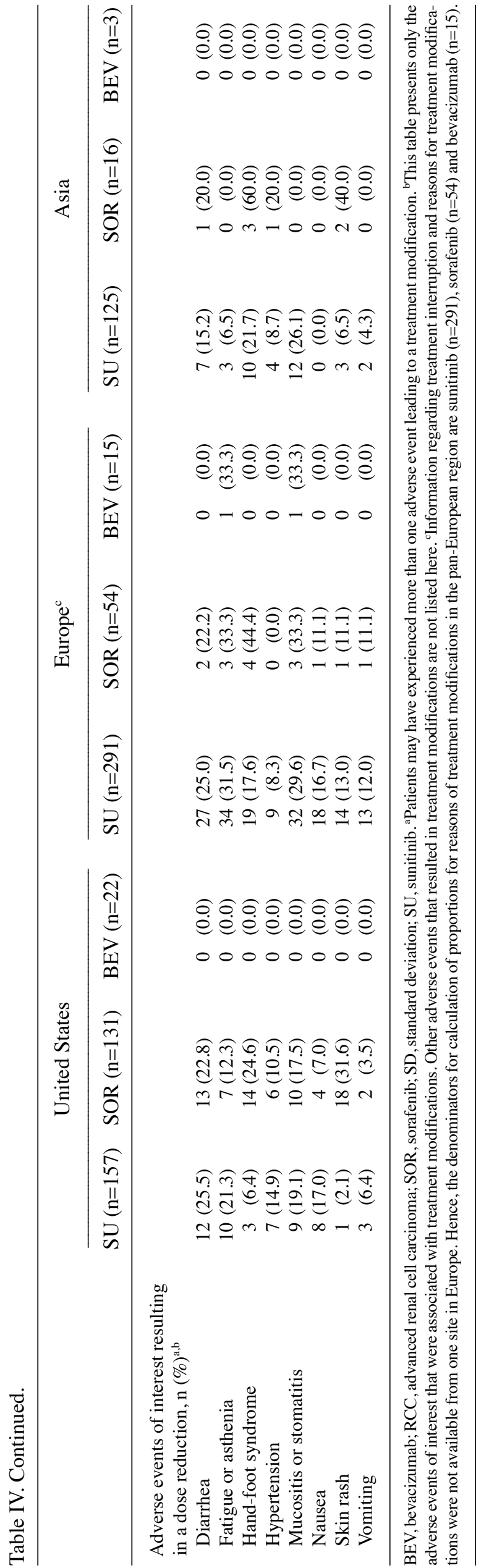

discontinued due to toxicity. A high proportion of patients in that study $(>75 \%)$ experienced fatigue, anorexia and hand-foot syndrome. In the Korean study by Hwang et al (15), 29\% of sunitinib patients experienced a dose reduction. In the UK study by Ansari et al (16), 15\% of sunitinib patients experienced a dose discontinuation in their first cycle of treatment, and $75 \%$ experienced at least one dose reduction. Notably, the number of patients in the current study was several fold higher than the aforementioned observational studies.

Some disparities in study results between this study and other observational studies reported above may have occurred due to differences in treatment durations, frequency of patient visits where AEs are reported, incomplete or inadequate recording of AEs, and differences in practice patterns relative to management of AEs across countries. Differences in drug approval dates, affecting drug availability, could have affected practice patterns as well. Differences in healthcare should also be kept in mind while making comparisons across studies.

There are some limitations associated with this study. Since data collection for this study preceded marketing authorization for pazopanib in Europe, this study does not include information on patients receiving pazopanib as first-line treatment. Further, due to the small sample sizes in certain groups for some regions, especially bevacizumab in all regions and sorafenib in Asia, the findings reported are descriptive in nature.

This multi-country study provides evidence that AEs are common in patients with advanced RCC treated with angiogenesis inhibitors, and that these AEs often lead to treatment modifications in the real-world clinical setting. This real-world practice study suggests that management of toxicities associated with anti-angiogenic agents for the treatment of advanced RCC presents significant issues for treating physicians and patients. The findings from this study further underscore the continued need for novel tolerable treatment options for advanced RCC. Additionally, the results of this study show the potential benefits of use of observational studies to further understand real-world treatment patterns and outcomes, beyond information that may be available from other data sources.

\section{Acknowledgements}

This study was funded by GlaxoSmithKline (GSK), Collegeville, PA. The funding from GSK was not contingent upon the study results. GSK participated in the study design, results interpretation and manuscript preparation as reflected in the authorship by GSK employees, M.P.N., J.R.D. and F.M.. A molecule with similar mechanism of action developed by GSK (pazopanib) was recently approved by the United States Food and Drug Administration and European Medical Agency for treatment of patients with advanced renal cell carcinoma. The institutions where W.K.O., D.M., C.P., A.L., R.E., F.S., R.H., D.C., J.B., S.Y.R., J.-M.S., P.N., B.A.F., J.S., R.M., J.-H.A., J.W., Y.-H.C., Y.-C.O., P.D., C.-Y.H., J.M., P.-H.C. and C.-K.C. are, have received research funds from Analysis Group Inc. M.S.D. and C.K. are employees of Analysis Group Inc., which has received research grants from GSK. 


\section{References}

1. Cohen HT and McGovern FJ: Renal-cell carcinoma. N Engl J Med 353: 2477-2490, 2005.

2. Gitlitz BJ and Figlin RA: Cytokine-based therapy for metastatic renal cell cancer. Urol Clin North Am 30: 589-600, 2003.

3. Motzer RJ, Mazumdar M, Bacik J, Berg W, Amsterdam A and Ferrara J: Survival and prognostic stratification of 670 patients with advanced renal cell carcinoma. J Clin Oncol 17: 2530-2540, 1999.

4. Motzer RJ, Hutson TE, Tomczak P, et al: Overall survival and updated results for sunitinib compared with interferon alpha in patients with metastatic renal cell carcinoma. J Clin Oncol 27: 3584-3590, 2009.

5. Escudier BE, Eisen T, Stadler WM, et al: Sorafenib for treatment of renal cell carcinoma: final efficacy and safety results of the phase III treatment approaches in renal cancer global evaluation trial. J Clin Oncol 27: 3312-3318, 2009.

6. Escudier BE, Pluzanska A, Koralewski P, et al: Bevacizumab plus interferon alpha-2a for treatment of metastatic renal cell carcinoma: a randomized, double-blind phase III trial. Lancet 370: 2103-2111, 2007.

7. Gore ME, Szczylik C, Porta C, et al: Safety and efficacy of sunitinib for metastatic renal-cell carcinoma: an expandedaccess trial. Lancet Oncol 10: 757-763, 2009.

8. Stadler WM, Figlin RA, McDermott DF, et al: Safety and efficacy results of the advanced renal cell carcinoma sorafenib expanded access program in North America. Cancer 116 : 1272-1280, 2010.

9. Food and Drug Administration. Available at http://www. accessdata.fda.gov/drugsatfda_docs/appletter/2005/021923ltr. pdf. Accessed December 19, 2011.

10. Bayer and Onyx press release: 'Nexavar approved by European Commission for Treatment of Advanced Kidney Cancer'. July 24,2006

11. Zhang H, Dong B, Lu JJ, et al: Efficacy of sorafenib on metastatic renal cell carcinoma in Asian patients: results from a multicenter study. BMC Cancer 9: 249, 2009.

12. National Cancer Institute. Available at: http://www.cancer. gov/cancertopics/druginfo/fda-sunitinib-malate. Accessed December 19, 2011.

13. Pfizer press release: 'Pfizer's Sutent ${ }^{\circledR}$ is Granted Full Marketing Authorization for First-Line Use in Advanced Kidney Cancer in European Union'. January 18, 2007.
14. Hong MH, Kim HS, Kim C, et al: Treatment outcomes of sunitinib treatment in advanced renal cell carcinoma patients: a single cancer center experience in Korea. Cancer Res Treat 41: 67-72, 2009.

15. Hwang E, Lee HJ, Sul CK and Lim JS: Efficacy and safety of sunitinib on metastatic renal cell carcinoma: a single institution experience. Korean J Urol 51: 450-455, 2010.

16. Ansari J, Fatima A, Fernando K, Collins S, James ND and Porfiri E: Sunitinib in patients with metastatic renal cell carcinoma: Birmigham experience. Oncol Rep 24: 507-510, 2010.

17. Yoo C, Kim JE, Lee JE, Ahn JH, Lee DH, Lee JS, et al: The efficacy and safety of sunitinib in Korean patients with advanced renal cell carcinoma: high incidence of toxicity leads to frequent dose reduction. Jpn J Clin Oncol 40: 980-985, 2010.

18. Ueda T, Imamura Y, Komaru A, et al: Treatment outcomes of sorafenib for first line or cytokinerefractory advanced renal cell carcinoma in Japanese patients. Int J Urol 17: 811-816, 2010.

19. Porta C, Paglino C, Imarisio I, et al: Safety and treatment patterns of multikinase inhibitors in patients with metastatic renal cell carcinoma at a tertiary oncology center in Italy. BMC Cancer 11: 105, 2011.

20. Feinberg BA, Jolly P, Wang S, et al: Safety and treatment patterns of angiogenesis inhibitors in patients with metastatic renal cell carcinoma: evidence from US community oncology clinics. Med Oncol 29: 786-794, 2012.

21. Choueiri TK, Duh MS, Clement J, et al: Angiogenesis inhibitor therapies for metastatic renal cell carcinoma: effectiveness, safety and treatment patterns in clinical practice based on medical chart review. BJU Int 105: 1247-1254, 2010.

22. Common Terminology Criteria for Adverse Events (CTCAE) version 3.0. Available: http://ctep.cancer.gov/protocolDevelopment/electronic_applications/docs/ctcaev3.pdf. Accessed: November, 2011.

23. Houk BE, Bello CL, Kang D, et al: A population pharmacokinetic meta-analysis of sunitinib malate (SU11248) and its primary metabolite (SU12662) in healthy volunteers and oncology patients. Clin Cancer Res 15: 2497-2506, 2009.

24. Escudier B, Bellmunt J, Negrier S, et al: Phase III trial of bevacizumab plus interferon alfa-2a in patients with metastatic renal cell carcinoma (AVOREN): final analysis of overall survival. J Clin Oncol 28: 2144-2150, 2010.

25. Rini BI, Halabi S, Rosenberg JE, et al: Phase III trial of bevacizumab plus interferon alfa versus interferon alfa monotherapy in patients with metastatic renal cell carcinoma: final results of CALGB 90206. Clin Oncol 28: 2137-2143, 2010. 